\title{
The role of diffusion tensor imaging for the assessment of liver fibrosis and inflammation in chronic viral hepatitis: A preliminary study
}

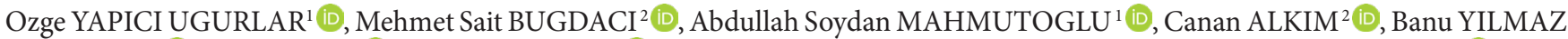 \\ OZGUVEN $^{3}$ (D), Alper OZEL ${ }^{1}$ (D), Muzaffer BASAK ${ }^{1}$ (D), Yasin AYKUT ${ }^{1}$ (iD, Huseyin OZKURT ${ }^{1}$ (D), Sukru Mehmet ERTURK ${ }^{1}$ id

\begin{abstract}
Radiology Clinic, Şişli Etfal Training and Research Hospital, Istanbul, Turkey.
Gastroenterology Clinic, Şişli Etfal Training and Research Hospital, Istanbul, Turkey.
\end{abstract} \\ 3 Pathology, Şişli Etfal Training and Research Hospital, Istanbul, Turkey.
}

Corresponding Author: Sukru Mehmet ERTURK

Submitted: $09.01 .2020 \quad$ Accepted: 02.03 .2020

E-mail: smerturk@gmail.com

\section{ABSTRACT}

Objective: To evaluate the role of magnetic resonance (MR) diffusion tensor imaging (DTI) in the assessment of liver fibrosis and inflammation in chronic viral hepatitis by measuring apparent diffusion coefficient (ADC) and fractional anisotropy (Fa) values. Material and Methods: Twenty-seven patients (5 women and 22 men;mean age39.8 \pm 11.3 ) with hepatic fibrosis were included in our study. The relationship between ADC, Fa values and histological activity index (HAI) score were evaluated using Spearman's correlation coefficient. Patients were subgrouped as group A (fibrosis score 1) and group B (fibrosis score 3). The mean ADC and Fa values of groups A and B were compared using Student's t-test. To evaluate the use of ADC and Fa values in distinguishing group A from group B, ROC analysis was applied; sensitivities and specificities were calculated.

Results: Fa values correlated with HAI scores significantly $(r=0.397, p<0.05)$ The difference was significant between the mean ADC values (group A:1.46 \pm 0.191x103mm2/s; group B:1.105 \pm 0.141x10-3 mm2/s; $<<0.001$ ) and Fa values (group A: 0.473 \pm 0.091 ; group B: $0.643 \pm 0.007 ; \mathrm{p}<0.001$ ) between the groups. For distinguishing group A from group B, ADC had a sensitivity of $81.8 \%$ and a specificity of $93.8 \%$. The sensitivity and specificity of Fa were $90.9 \%$ and $100 \%$, respectively.

Conclusion: Diffusion tensor imaging may play a role in the evaluation of fibrosis and HAI scores in patients with liver fibrosis.

Keywords: Hepatitis, Liver fibrosis, Diffusion tensor imaging.

\section{INTRODUCTION}

The detection of hepatic fibrosis and inflammation in patients with chronic viral hepatitis is crucial for planning the treatment and follow-up strategies which will affect the long-term prognosis [1]. Anti-viral treatment can eradicate the infection, increase patient survival and reduce the need for liver transplantation [1]. Antiviral treatment is indicated in patients with moderate to advanced stages of fibrosis. The score of fibrosis and HAI scores are determined by histopathologic evaluation obtained after liver biopsy. However, liver biopsy has some limitations such as its complication rate [1-5\%] and high cost $[2,3]$. Thus, noninvasive methods are needed to diagnose and score the liver fibrosis.

Over the last years, investigations on magnetic resonance (MR) diffusion weighted imaging (DWI) have been carried out to provide a noninvasive way of detection and quantification of liver fibrosis [1,4-10]. The microscopic mobility of the water protons is the aspect that DWI relies on. Apparent diffusion coefficient $(\mathrm{ADC})$ indicates the movement of water molecules in a tissue [4]. The fibrotic changes cause architectural distortions of the liver parenchyma and lead to the diffusion restriction of water molecules. As expected, ADC value decreases as the degree of fibrosis increases in the tissue [1,4-10].

Unlike DWI, diffusion tensor imaging (DTI) can be helpful for evaluating anisotropic properties of tissues since, it allows the analysis of diffusion along multiple directions [11]. By employing DTI, not only ADC but also fractional anisotropy ( $\mathrm{Fa}$ ) values can be calculated. Fa values reveal the fraction of anisotropic diffusion to total diffusion. There is very limited research on the utility of DTI in staging of fibrosis. To our knowledge, only one study has examined the usefulness of DTI in regard to liver fibrosis [1].

Our hypothesis is that the fibrotic and inflammatory changes encountered during the course of chronic hepatitis will not only cause restricted diffusion, but will also cause an increase in the fraction of anisotropic diffusion which will lead to a decrease in $\mathrm{ADC}$ and increase in Fa values obtained by DTI. 
The objective of this study is to test the hypothesis by correlating both ADC and Fa values with the degree of fibrosis and histological activity index (HAI) scores in patients with chronic viral hepatitis.

\section{MATERIALS and METHODS}

\section{Patients and Histopathologic Analysis}

Institutional review board approval was obtained for this retrospective single-institution study. The data bank of the gastroenterology clinic was searched to find patients who had liver biopsy due to either chronic hepatitis B or C. Among those patients, the ones who underwent MR-DTI were included in the study between April 2009 and June 2010. There were a total of 27 patients ( 5 women and 22 men; mean age $=39.8 \pm 11.3$ ). The mean time interval between biopsy date and imaging was 177.2 \pm 232.4 days. Twenty-two patients had chronic hepatitis B and five patients had chronic hepatitis C. The HAI score and scores of fibrosis were documented for each patient according to histopathology reports of the liver biopsies. In 1981, HAI scoring system was described by Knodell et al. In this scoring system, necroinflammatory activity of the liver biopsy specimens are formulated and scored between 0 and 18, by combining periportal necrosis and inflammation score (between 0 and 10), lobular necrosis and inflammation score (between 0 and 4), and portal inflammation score (between 0 and 4) [12,13]. In our study, 26 patients had HAI scores calculated according to Knodell system; median HAI score was 7 (range:2-11) (one patient had a fibrosis score, but no HAI score was calculated according to his liver biopsy report ).

Fibrosis in the HAI system is scored as 1, 3, or 4, with 1 indicating portal fibrosis only, 3 indicating bridging fibrosis, and 4 indicating cirrhosis [13]. This discontinuous scale (2 is missing) is used to allow a clear separation of mild (score 1) from extensive (score 3) fibrosis [13]. In our study, 16 patients had fibrosis score 1 , and 11 patients had fibrosis score 3 (one patient had a fibrosis score, but no HAI score was calculated according to the HAI system). We were not able to include any patients with fibrosis score 4 (cirrhosis) in this retrospective study; the gastroenterologists at our institution were reluctant to refer cirrhotic patients (who were diagnosed according to clinical, laboratory and liver imaging findings) to liver biopsy due to high risk of biopsy-related complications.

\section{MR Imaging}

All MR imaging examinations were performed using a 1.5Tesla Scanner (GE Healthcare, Waukesha, WI ). In addition to the conventional MR imaging protocol, an axial breath-hold, singleshot gradient echo planar DTI sequence covering the whole liver was acquired using the following parameters: Matrix: 256x160, Acquisition time: 3:04 (min:sec), TE: $89.4 \mathrm{msec}$, TR: 6125.0 msec, Bandwith: $31.25 \mathrm{kHz}$, number of excitations: 4.0, field of view: 48 , Slide thickness: $8.0 \mathrm{~mm}$, Spacing: $1.0 \mathrm{~mm}$, b-value: $1000 \mathrm{sec} / \mathrm{mm} 2$, number of diffusion directions: 6. Spectro Spatial RF pulse was used to reduce chemical shift artifacts.
Whereas atypical conventional DWI sequence employs three diffusion gradients in three orthogonal directions (frequencyencoding ( $\mathrm{x})$, phase-encoding $(\mathrm{y})$, and section-select $(\mathrm{z})$, our DTI sequence employs six directions $(x, y, z, x y, y z$ and $x z)$ as described previously [1].

\section{Image Analysis}

Two radiologists who were blinded to fibrosis scores and HAI scores reviewed all images, independently. ADC and Fa maps were calculated on a remote Workstation (GE, Advantage Windows Workstation, Milwaukee, WI). Three regions of interest (ROIs) with equal diameters (approximately $1 \mathrm{~cm}$ ) were placed in the right lobe of the liver away from intrahepatic vasculature.

\section{Statistical Analysis}

The reports of two radiologists who measured ADC and $\mathrm{Fa}$ values were evaluated using Bland-Altman statistic. For further analysis, their measurements were averaged. The mean ADC and $\mathrm{Fa}$ values were calculated first for all patients and then separately for groups A (fibrosis score 1) and B (fibrosis score 3 ).

The relationship between ADC and Fa values in our patients was evaluated using Pearson's correlation. The relationship between HAI scores and ADC or Fa values in the whole group was evaluated using Pearson's correlation. Correlation coefficients $\geq 0.7,>0.5$ to $<0.69,>0.3$ to $<0.49,>0.1$ to $<0.29$, and $>0.01$ to $<0.09$ were interpreted as indicators of very strong, substantial, moderate, low, and negligible associations, respectively [14]. P values $<0.05$ were considered statistically significant.

Then, patients were subgrouped as group A (fibrosis score 1) and group B (fibrosis score 3 ) according to the results of histopathological analysis obtained by liver biopsy. The mean $\mathrm{ADC}, \mathrm{Fa}$ ages and values of patients in group A (fibrosis score 1 ) and B (fibrosis score 3 ) were compared using Student's t-test. Regarding the gender and the hepatitis type, groups were compared using Fisher exact test. P values $<0.05$ were considered statistically significant.

To evaluate the use of ADC and Fa values in distinguishing patients with fibrosis score 1 (group A) from those with fibrosis score 3 (group B), a ROC analysis was applied. The areas under the curve and cut-off values were calculated. Sensitivities and specificities with $95 \%$ confidence intervals (CIs) were calculated accordingly.

\section{RESULTS}

There was a good agreement between the two radiologists for measuring both ADC and Fa values according to Bland-Altman analysis (Figure1). Figure 2 demonstrates representative MR images of a patient with score 3 fibrosis and a HAI score of 10 . 

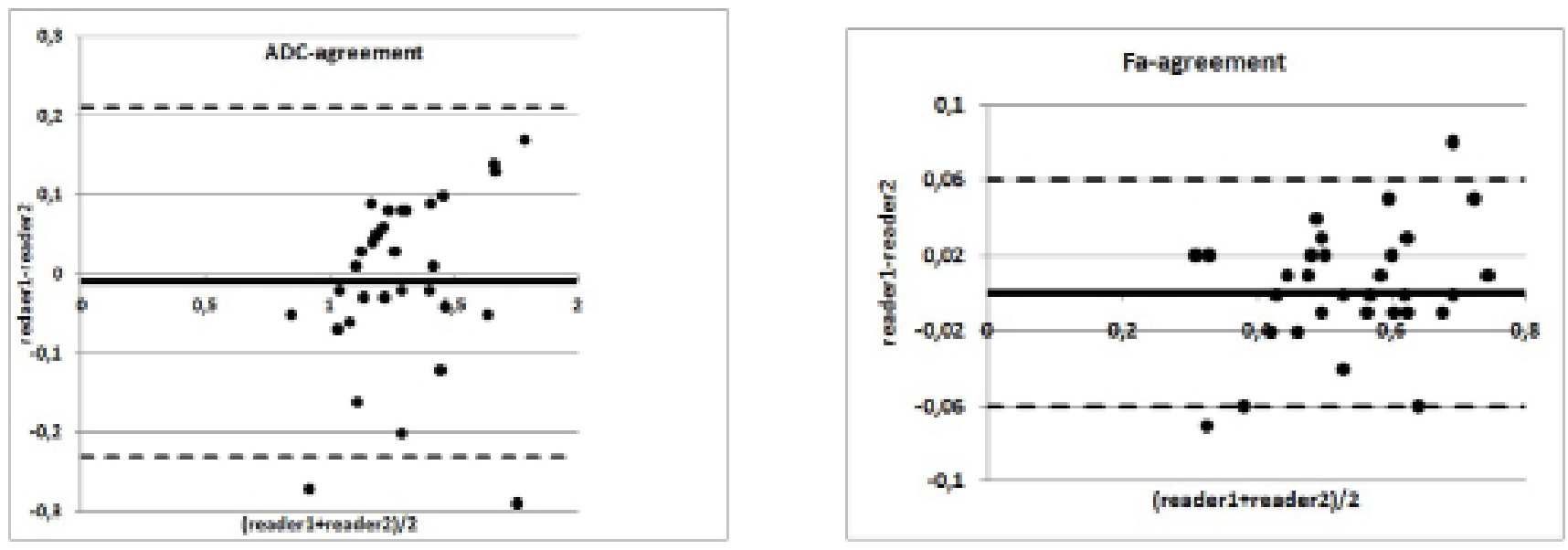

Figure 1. Graphs show results of Bland-Altman test regarding the interobserver agreement on ADC (a) and Fa (b) measurements. The solid line represents mean difference, and the dashed lines represent limits of agreement $(d \pm 2 S D)$.

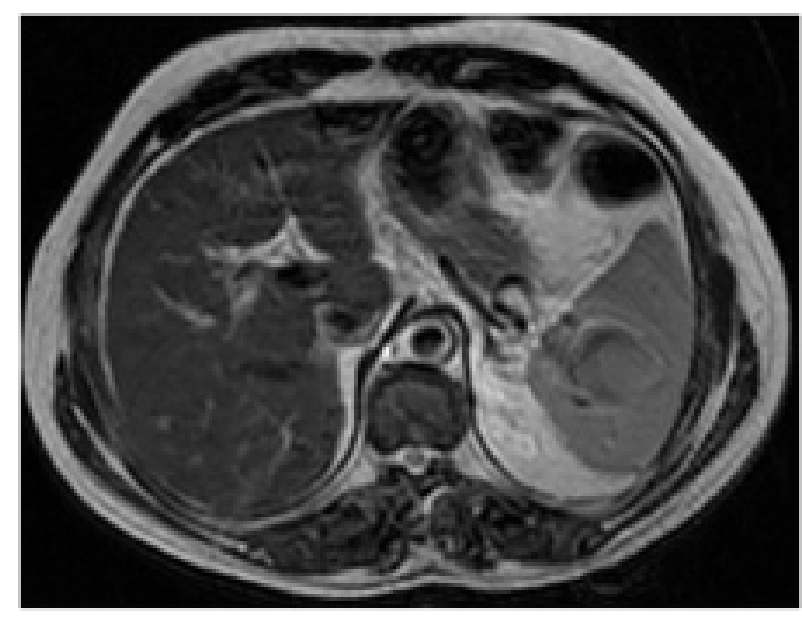

a)

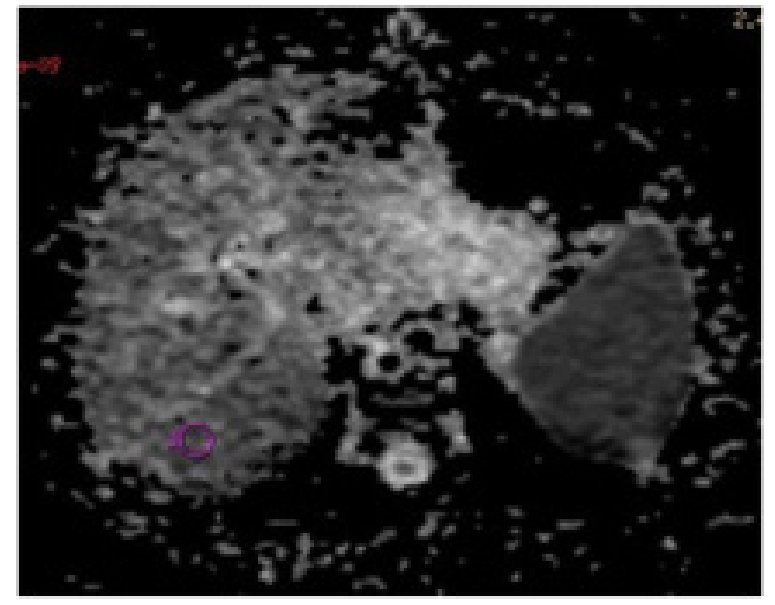

b)

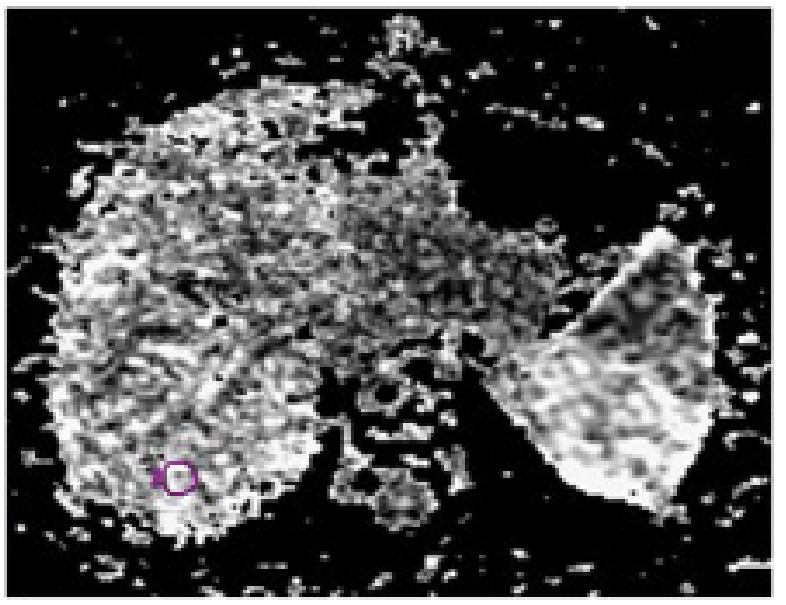

c)

Figure 2. T2-weighted image (a), ADC map (b) and Fa map (c) of a patient with score 3 fibrosis. His HAI score was 10. 
In the whole group, mean $\mathrm{ADC}$ and Fa values were $1.31 \pm 0.24$ $\mathrm{x} 10^{-3} \mathrm{~mm}^{2} / \mathrm{s}$ and $0.54 \pm 0.12$, respectively. There was a negative, very strong and significant $(\mathrm{r}=-0.864, \mathrm{p}<0.00000001)$ correlation between ADC and Fa values (Table I).

Table I. Gender, mean age, number of the patients according to the type of the chronic viral hepatitis, mean ADC and Fa values are given for fibrosis score 1 (group A) and fibrosis score 3 (group B) patients.

\begin{tabular}{|l|c|c|c|}
\hline & Fibrosis Score 1 (group A) & $\begin{array}{c}\text { Fibrosis Score 3 } \\
\text { (group B) }\end{array}$ & p Value \\
\hline Gender & 2 female, 14 male & 3 female, 8 male & $>0.1$ \\
\hline $\begin{array}{l}\text { Mean age } \pm \text { SD } \\
\text { (years) }\end{array}$ & $35.75 \pm 10.74$ & $45.64 \pm 8.60$ & $<0.05$ \\
\hline Hepatitis type & $\begin{array}{c}14 \text { hepatitis B, } \\
2 \text { hepatitis C }\end{array}$ & $\begin{array}{c}8 \text { hepatitis B, } \\
3 \text { hepatitis C }\end{array}$ & $>0.1$ \\
\hline $\begin{array}{l}\text { Mean ADC } \\
\text { value } \pm \text { SD }\end{array}$ & $1.46 \pm 0.191 \times 10^{-3} \mathrm{~mm}^{2} / \mathrm{s}$ & $\begin{array}{c}1.105 \pm 0.141 \times 10^{-3} \\
\mathrm{~mm}^{2} / \mathrm{s}\end{array}$ & $<0.001$ \\
\hline $\begin{array}{l}\text { Mean FA value } \\
\pm \text { SD }\end{array}$ & $0.463 \pm 0.08$ & $0.644 \pm 0.07$ & $<0.001$ \\
\hline
\end{tabular}

The correlation between ADC values and HAI scores was negative, moderate and not significant $(\mathrm{r}=-0.34, \mathrm{p}=0.088)$. The correlation between $\mathrm{Fa}$ values and HAI scores was positive, moderate and significant $(\mathrm{r}=0.397, \mathrm{p}=0.045)$. The mean ADC values in patients with fibrosis score 1 (group A) and in patients with fibrosis score 3 (group B) were $1.46 \pm 0.191 \times 10^{-3} \mathrm{~mm}^{2} / \mathrm{s}$ and $1.105 \pm 0.141 \times 10^{-3} \mathrm{~mm}^{2} / \mathrm{s}$, respectively (Student's t-test, $\mathrm{p}<0.001$ ). The mean $\mathrm{Fa}$ values in patients with fibrosis score 1(group A) and score 3 (group B) were $0.463 \pm 0.08$ and $0.644 \pm 0.07$, respectively, (Student's t-test, $\mathrm{p}<0.001$ ). ADC value had an area under the curve of 0.949 . Using $1.22 \times 10^{-3} \mathrm{~mm}^{2} / \mathrm{s}$ as the cut-off value, the sensitivity was $81.8 \%(9 / 11 ; 95 \% \mathrm{CI}=52.3 \%-94.9 \%)$ and the specificity was $93.8 \%(15 / 16 ; 95 \% \mathrm{CI}=71.7 \%-98.9 \%) . \mathrm{Fa}$ value had an area under the curve of 0.969 . Using 0.59 as the cut-off value the sensitivity was $90.9 \%(10 / 11 ; 95 \% \mathrm{CI}=62.3 \%$ $98.4 \%)$ and the specificity was $100 \%(16 / 16$; $95 \% \mathrm{CI}=80.6 \%$ $100 \%)$.

\section{DISCUSSION}

In our preliminary study, we aimed to find a correlation between Fa values, obtained by DTI with HAI scores and/or fibrosis scores obtained by histopathologic evaluation of liver biopsy specimens of patients with chronic viral hepatitis. We found out that Fa values correlate moderately and significantly with HAI scores. Since, severe degrees of inflammatory activity predict worsening of hepatic fibrosis and constitute an indication for therapy independent of the current level of fibrosis [13], this finding may play an important role in the follow-up of patients with chronic hepatitis. In distinguishing patients with fibrosis score 1 from those with fibrosis score 3, Fa and ADC values can be used successfully. The performance of Fa values was slightly better as reflected in Az values obtained through ROC analysis. This is also important, since a clear separation of patients with fibrosis score 1 from those with fibrosis score 3 is crucial in terms of clinical decision making $[13,15]$.

Although, the normal liver parenchyma exhibits anisotropic diffusion, our hypothesis was that the inflammation and fibrotic process encountered during the course of chronic hepatitis would cause further anisotropy. Our results show that this hypothesis might be true. Thus, in addition to ADC values, Fa values can also be used in the follow-up of the patients with hepatic fibrosis due to chronic hepatitis. Our results show that Fa values can be employed especially to monitor the histopathological activity.

There are studies trying to correlate ADC values with HAI scores and fibrosis scores using DWI $[7,10]$. In these studies, a negative correlation was reported between ADC values and fibrosis scores and HAI scores. Our results are parallel to the results of those studies. Nevertheless, the major difference between our study and the former ones is the employment of DTI instead of DWI. There are a few studies trying to employ DTI in the diagnosis of liver fibrosis and inflammation $[1,16]$. Taouli et al., in their pioneer study, reported a sensitivity of $78.3 \%$ and a specificity of $69.2 \%$ in distinguishing patients with fibrosis stage 1 from the patients with a higher fibrosis stage using an ADC value of 1.36 as cut-off [1]. They also reported that they did not take the advantage of calculating anisotropy with DTI, since it was previously shown that the liver has a near isotropic diffusion. Very recently, Tosun et al., reported in their study that $\mathrm{ADC}$ values showed a trend toward lower values and Fa values showed a trend toward higher values with increasing fibrotic stage, however, without statistically significant correlation [16]. They also reported that $\mathrm{ADC}$ values showed a trend toward lower values and $\mathrm{Fa}$ values showed a trend toward higher values with increasing inflammatory score. Although, our results are parallel to those of Tosun et al's., we need to note that the histopathological evaluation method we employed was different. We determined HAI scores and fibrosis scores according to Knodell et al, although Tosun et al. used METAVIR system.

Furthermore, we tried to correlate HAI scores with ADC and Fa values, not the inflammation scores. Our results showed that using Fa values in the diagnosis of liver fibrosis and inflammation might have an additional advantage. In our study, using a $\mathrm{Fa}$ value of 0.59 as cut-off value, the sensitivity and specificity of DTI in distinguishing patients with fibrosis score 1 from the patients with fibrosis score 3 were $90.9 \%$ and $100 \%$, respectively. Although, liver biopsy is the gold standard in the diagnosis and the most valuable method used in the management of patients with chronic hepatitis, being an invasive procedure it may have minor to major complications. Furthermore, it may not be easily accepted by patients. Therefore alternative non-invasive and reliable approaches to diagnose and follow-up of liver fibrosis are needed. In our understanding DTI might be a promising tool in this regard. ADC and Fa values are biomarkers that need to be further evaluated.

Nevertheless, our study has several limitations. First, it is a retrospective study that has intrinsic shortcomings. On the 
contrary of a prospective study, the medical management was not changed according to neither Fa nor ADC values for none of the patients. Second, as mentioned before, because this is a retrospective study, there was a relatively long time interval between the histopathological evaluation of the liver biopsies and the MR imaging of the patients. Despite this fact, we could still get statistically significant results. Third, we were not able to include any patients with fibrosis score 4 in this study. Nevertheless, as we mentioned above, a clear separation of patients with fibrosis score 1 from those with fibrosis score 3 is crucial in terms of clinical decision making $[13,15]$. Patients with score 4 fibrosis (cirrhosis) are typically diagnosed using laboratory and conventional imaging findings and do not constitute a real diagnostic challenge for gastroenterologists. Fourth, our study population was relatively small. However, we still reached statistically significant results.

In conclusion, Fa and ADC values obtained by employing DTI might be promising quantitative biomarkers that can be used in the management of patients with liver fibrosis due to chronic hepatitis. Prospective studies with larger patient populations are needed to support our preliminary results.

\section{Compliance with Ethical Standards}

Ethical Approval: This study was approved by Şişli Etfal Training and Research Hospital Ethics Committee. (Protocol number:401). All methods were performed in accordance with the relevant guidelines regulations.

Funding: This research received no funding.

Conflict of Interest: The authors declare that they have no conflict of interest.

Authors' Contribution: Concept: OYU, ASM and SME, Data collection and planning: MSB, Data collection and analysis: CA, BYO, YA, Analysis and evaluation: AO, MB, Literature review and planning: HO, Critical review: SME, Final approval: all the authors.

\section{REFERENCES}

[1] Taouli B, Chouli M, Martin AJ, et al. Chronic hepatitis: role of diffusion weighted imaging and diffusion tensor imaging for the diagnosis of liver fibrosis and inflammation. J Magn Reson Imaging 2008; 28:89-95. doi.org/10.1002/jmri.21227.

[2] Annet L, Peeters F, Abarca-Quinones J, Leclercq I, Moulin P, Van Beers BE. Assessment of diffusion-weighted MR imaging in liver fibrosis. J Magn Reson Imaging 2007; 25:122-8. doi:10.1002/jmri.20771.

[3] Aguirre DA, Behling CA, Alpert E, Hassanein TI, Sirlin CB. Liver fibrosis: noninvasive diagnosis with double contrast material-enhanced MR imaging. Radiology 2006;239:425-37. doi.org/10.1148/radiol. 239.205.0505.

[4] Bonekamp S, Torbenson MS, Kamel IR. Diffusion-weighted magnetic resonance imaging for the staging of liver fibrosis. J Clin Gastroenterol 2011;45:885-92. doi: 10.1097/ MCG.0b013e318223bd2c.

[5] Sandrasegaran K, Fatih M, Akisik FM, et al. Value of diffusionweighted MRI for assessing liver fibrosis and cirrhosis. Am J Roentgenol 2009;193:1556-60. doi:10.2214/AJR.09.2436

[6] Koinuma MN, Ohashi I, Hanafusa K, et al. Apparent diffusion coefficient measurements with diffusion-weighted magnetic resonance imaging for evaluation of hepatic fibrosis. J Magn Reson Imaging 2005;22:80-5.doi. org/10.1002/jmri.20344.

[7] 7. Taouli B, Toila AJ, Losada M, et al. Diffusion-weighted MRI for quantification of liver fibrosis: preliminary experience. Am J Roentgenol 2007;189:799-806. doi:10.2214/AJR.07.2086.

[8] 8. Girometti R, Furlan A, Bazzocchi M, etal. Diffusionweighted MRI in evaluating liver fibrosis: a feasibility study in cirrhotic patients. Radiol Med 2007;112:394-408. doi: 10.1007/ s11547.007.0149-1.

[9] Soylu A, Kılıçkesmez Ö, Poturoğlu Ş, et.al. Utility of diffusionweighted MRI for assessing liver fibrosis in patients with chronic active hepatitis. Diagn Interv Radiol 2010; 16:204-8. doi: 10.4261/1305-3825.DIR. 2810-09.1.

[10] Bakan AA, Inci E, Bakan S, Gokturk S, Cimilli T. Utility of diffusion-weighted imaging in the evaluation of liver fibrosis. EurRadiol 2012;22: 682-7. doi:10.1007/s00330.011.2295-z.

[11] Taouli B, Martin AJ, Qayyum A, Merriman RB, et.al. Parallel imaging and diffusion tensor imaging for diffusion-weighted MRI of the liver: Preliminary experience in healthy volunteers. AJR Am J Roentgenol 2004;183:677-80. doi: 10.2214/ ajr.183.3.1830677.

[12] Knodell RG, Ishak KG, Black WC, et al. Formulation and application of a numerical scoring system for assessing histological activity in asymptomatic chronic active hepatitis. Hepatology 1981;1:431-5. doi.org/ 10.1002/hep.184.001.0511.

[13] Marcellin P, Asselah T, Boyer N. Fibrosis and disease progression in hepatitis C. Hepatology 2002; 36:47-56. doi: 10.1053/jhep.2002.36993.

[14] Davis JA. Elementary survey analysis. Englewood Cliffs, NJ: Prentice-Hall,1971:49.

[15] Shiffman ML, Benhamou Y. Patients with HCV and F1 and F2 fibrosis stage:treat now or wait? Liver Int 2013; 33:105-10. doi: 10.1111/liv.12066.

[16] Tosun M, Inan N, Sarisoy HT, et.al. Diagnostic performance of conventional diffusion weighted imaging and diffusion tensor imaging for the liver fibrosis and inflammation. Eur J Radiol. 2013;82(2):203-7. doi: 10.1016/j.ejrad.2012.09.009. 